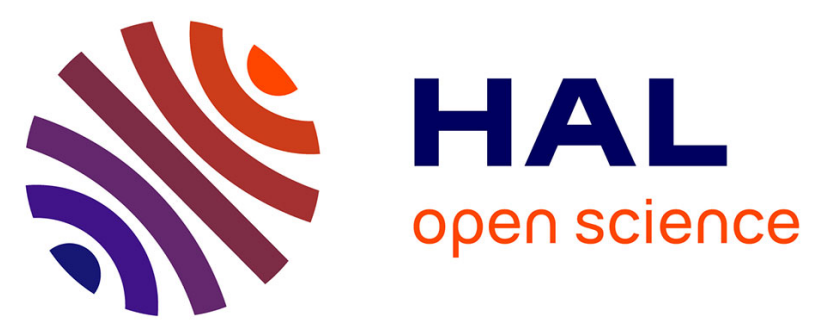

\title{
Angular Resolution Limit for Vector-Sensor Arrays: Detection and Information Theory Approaches
}

Dinh Thang Vu, Mohammed Nabil El Korso, Remy Boyer, Alexandre Renaux, Sylvie Marcos

\section{- To cite this version:}

Dinh Thang Vu, Mohammed Nabil El Korso, Remy Boyer, Alexandre Renaux, Sylvie Marcos. Angular Resolution Limit for Vector-Sensor Arrays: Detection and Information Theory Approaches. IEEE International Workshop on Statistical Signal Processing - invited article, 2011, Nice, France. pp.9-12. hal-00577272

\section{HAL Id: hal-00577272 \\ https://hal.science/hal-00577272}

Submitted on 17 Mar 2011

HAL is a multi-disciplinary open access archive for the deposit and dissemination of scientific research documents, whether they are published or not. The documents may come from teaching and research institutions in France or abroad, or from public or private research centers.
L'archive ouverte pluridisciplinaire HAL, est destinée au dépôt et à la diffusion de documents scientifiques de niveau recherche, publiés ou non, émanant des établissements d'enseignement et de recherche français ou étrangers, des laboratoires publics ou privés.

\section{(1)(1) $\$(0)$}

Distributed under a Creative Commons Attribution - NonCommercial - ShareAlikel 4.0 


\title{
ANGULAR RESOLUTION LIMIT FOR VECTOR-SENSOR ARRAYS: DETECTION AND INFORMATION THEORY APPROACHES
}

\author{
Dinh Thang VU, Mohammed Nabil EL KORSO, Rémy BOYER, Alexandre RENAUX and Sylvie MARCOS
}

\author{
Laboratoire des Signaux et Systèmes (L2S) \\ Université Paris-Sud XI (UPS), CNRS, SUPELEC \\ Gif-Sur-Yvette, France
}

\begin{abstract}
The Angular Resolution Limit (ARL) on resolving two closely spaced polarized sources using vector-sensor arrays is considered in this paper. The proposed method is based on the information theory. In particular, the Stein's lemma provides, asymptotically, a link between the probability of false alarm and the relative entropy between two hypothesis of a given statistical binary test. We show that the relative entropy can be approximated by a quadratic function in the ARL. This property allows us to derive and analyze a closed-form expression of the ARL. To illustrate the interest of our approach the ARL, in the sense of the detection theory, is also derived. Finally, we show that the ARL is only sensitive to the norm of the polarization state vector and not to the particular values of the polarization parameters.
\end{abstract}

Index Terms - Angular resolution limit, polarized source localization, distance measure, information theory, detection theory.

\section{INTRODUCTION}

The Direction-Of-Arrivals (DOA) estimation for polarized sources based on the vector-sensor arrays have been largely investigated in the last decade. In [1], the authors considered the problem of source localization in a multipath environment by using a vector-sensor array consisting of three electric and three magnetic dipoles. In [2], a tensorial version of the MUSIC algorithm for vector-sensor arrays was presented. On the other hand, the polarized seismic wave estimation was considered in [3]. Whereas, in [4], closed form expressions of Cramér-Rao Bound (CRB) have been derived.

In array processing, the Angular Resolution Limit (ARL) characterizes the minimum angular separation to resolve two closely spaced sources. In the literature, there are three approaches to obtain the ARL. (1) The first approach based on the estimation accuracy. In this way, Smith [5] proposed the following criterion based on the CRB: two signals are resolvable if the separation between the two DOAs $\theta_{1}$ and $\theta_{2}$ is less than the standard deviation of the separation estimation. Consequently, the ARL in the Smith sense is defined as the angular separation between the parameters of interest that is equal to the standard deviation of the angular separation. Furthermore, in [6], the extension of the ARL in the case of multiple parameters per signal based on Smith criteria was presented. (2) The second approach based on the concept of the mean null spectrum [7]. This approach is quite intuitive but is only relevant to a specific high-resolution algorithm. (3) The third approach based on

This project is funded by both the Digiteo Research Park and the Region Ile-de-France. detection theory. In [8], the ARL based on the Minimum Probability of Error (MPE) for the deterministic signals is investigated. The authors used the first order Taylor expansion of the MPE to derive the ARL. Whereas, in [9] Liu and Nehorai have defined the statistical angular resolution limit using the asymptotic equivalence of the Generalized Likelihood Ratio Test (GLRT). On the other hand, Sharman and Milanfard [10] derived the frequency resolution limit in the spectral analysis using the GLRT.

In this paper, we propose a new approach of the ARL based on the information theory, and more specifically on the Stein's lemma [11]. The Stein's lemma links the false alarm probability $\left(P_{f a}\right)$ resulted from the Neyman-Pearson decision criterion to the relative entropy (also called Kullback-Leiber pseudo-distance). As the relative entropy can be approximated by a quadratic function in the ARL, it is possible to determine the ARL by this way. To illustrate our approach, the ARL is derived in the Neyman-Pearson sense in the context of the Bayesian detection theory. Finally, we also compare our approach to the one presented by Amar and Weiss in reference [8].

\section{MODEL SETUP}

\subsection{Polarized signal model}

We consider the context of DOA estimation of two narrow-band polarized source signals using a linear two elements vector-sensor array. Without loss of generality, we assume that the array lays on the $O x$ axis of the Cartesian coordinate. The array consists of $N$ vector-sensors and the known positions of these vector-sensors in the array are given by the vector $\mathbf{d}=\left[d_{1} \ldots d_{N}\right]^{T}$. The two sources are assumed to be located in the far-field, deterministic, and coplanar with the array, i.e., the source elevations equal $\theta_{k}=\pi / 2$, $k=\{1,2\}^{2}$. For mathematical convenience, we consider the estimation of $u_{k}=\frac{2 \pi}{\lambda} \sin \phi_{k}$, where $\lambda$ denotes the wavelength.

We assume that the source polarization is constant in time and along the array. The polarization of the sources is characterized by the vector [2]

$$
\mathbf{p}(\rho, \varphi)=\left[\begin{array}{l}
{[\mathbf{p}(\rho, \varphi)]_{1}} \\
{[\mathbf{p}(\rho, \varphi)]_{2}}
\end{array}\right]=\frac{1}{\sqrt{\rho^{2}+1}}\left[\begin{array}{l}
1 \\
\rho e^{j \varphi}
\end{array}\right]
$$

where $\rho$ and $\varphi$ denote the amplitude ratio and the phase shift between the second component of the sensor and the first [2]. At the $t^{t h}$ snapshot, the output signal in the time domain at the $i^{t h}$ vectorsensor consists of two components (the model in frequency domain 
is available in [2])

$$
\begin{aligned}
& \hat{z}_{i}(t)=\sum_{k=1}^{2}[\mathbf{p}(\rho, \varphi)]_{1}\left[\mathbf{a}\left(u_{k}\right)\right]_{i} s_{k}(t)+\hat{n}_{i}(t) \\
& \breve{z}_{i}(t)=\sum_{k=1}^{2}[\mathbf{p}(\rho, \varphi)]_{2}\left[\mathbf{a}\left(u_{k}\right)\right]_{i} s_{k}(t)+\breve{n}_{i}(t)
\end{aligned}
$$

where $\mathbf{a}\left(u_{k}\right)=\left[\begin{array}{lll}e^{-j d_{1} u_{k}} & \ldots & e^{-j d_{N} u_{k}}\end{array}\right]^{T}$, and where $\breve{n}(t)$, $\hat{n}(t)$ denote the additive noises at the $t^{t h}$ snapshot, and $s_{k}(t)$ denotes the source signal of the $k^{t h}$ source at the $t^{t h}$ observation. We assume that $s_{1}(t) \neq s_{2}(t), \forall t$. The noises are assumed to be complex, circular, white Gaussian with zero mean and covariance matrix $\sigma^{2} \mathbf{I}$, i.e., $\mathbf{n} \sim \mathcal{C N}\left(\mathbf{0}, \sigma^{2} \mathbf{I}\right)$. Furthermore, we assumed the signal sequences of the two sources are deterministic with known sequences at the observer. Consequently, at the $t^{\text {th }}$ snapshot, the

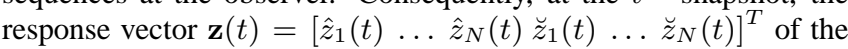
array is given by

$$
\mathbf{z}(t)=\sum_{k=1}^{2} \mathbf{b}\left(u_{k}\right) s_{k}(t)+\mathbf{n}(t)
$$

where $\mathbf{n}(t)$ denotes the noise vector and $\mathbf{b}\left(u_{k}\right)=\mathbf{p}(\rho, \varphi) \otimes \mathbf{a}\left(u_{k}\right)$ in which $\otimes$ stands for the Kronecker product.

\subsection{Bayesian MPE based binary hypothesis test}

We can now adopt the two detection hypotheses (see [8]): under hypothesis $\mathcal{H}_{0}$, the observer detects only a single signal, which is a combination of the two sources, and under $\mathcal{H}_{1}$, the observer detects two signals:

$$
\begin{cases}\mathcal{H}_{0}: & \mathbf{z}(t)=\mathbf{b}(\hat{u}(t)) \hat{s}(t)+\mathbf{n}(t) \\ \mathcal{H}_{1}: & \mathbf{z}(t)=\sum_{k=1}^{2} \mathbf{b}\left(u_{k}\right) s_{k}(t)+\mathbf{n}(t)\end{cases}
$$

where $\hat{u}(t)$ and $\hat{s}(t)$ denote the parameter and signal amplitude under $\mathcal{H}_{0}$, whereas the probability of error $P_{e}$ given by $P_{e}=$ $p\left(\mathcal{H}_{0}\right) P_{f a}+p\left(\mathcal{H}_{1}\right) P_{m}$, in which $P_{f a}$ and $P_{m}$ denote the probability of false alarm and the probability of miss, respectively, and where $p\left(\mathcal{H}_{0}\right), p\left(\mathcal{H}_{1}\right)$ denote the prior probability of the two hypotheses. Without loss of generality, we assume that $p\left(\mathcal{H}_{0}\right)=p\left(\mathcal{H}_{1}\right)=1 / 2$. Thus, setting $u_{c}=\frac{u_{1}+u_{2}}{2}$ and the ARL given by $\delta=u_{2}-u_{1}$, then, the values of $\hat{u}(t)$, and $\hat{s}(t)$ chosen according to the minimal probability of error (MPE) criteria are given by [8]:

$$
\begin{aligned}
& \hat{u}(t)=u_{c}+\gamma(t) \delta \\
& \hat{s}(t)=\frac{1}{N} \mathbf{b}^{H}(\hat{u}(t))\left(\mathbf{b}\left(u_{c}-\frac{\delta}{2}\right) s_{1}(t)+\mathbf{b}\left(u_{c}+\frac{\delta}{2}\right) s_{2}(t)\right)
\end{aligned}
$$

where $\gamma(t)=\frac{\left|s_{2}(t)\right|^{2}-\left|s_{1}(t)\right|^{2}}{2\left(\left|s_{2}(t)\right|^{2}+\left|s_{1}(t)\right|^{2}+2 \Re\left\{s_{1}^{*}(t) s_{2}(t)\right\}\right)}$. From the aforementioned assumption, it is clear that

$$
\begin{cases}\mathcal{H}_{0}: & \mathbf{z}(t) \sim \mathcal{C N}\left(\boldsymbol{\mu}_{0}(t), \sigma^{2} \mathbf{I}\right), \text { where } \boldsymbol{\mu}_{0}(t)=\mathbf{b}(\hat{u}(t)) \hat{s}(t), \\ \mathcal{H}_{1}: & \mathbf{z}(t) \sim \mathcal{C N}\left(\boldsymbol{\mu}_{1}(t), \sigma^{2} \mathbf{I}\right) \text { where } \boldsymbol{\mu}_{1}(t)=\sum_{k=1}^{2} \mathbf{b}\left(u_{k}\right) s_{k}(t) .\end{cases}
$$

\subsection{First-order Taylor expansions}

Assume that the polarization parameters are all equal ${ }^{1}$, i.e. $\rho_{1}=$ $\rho_{2}=\rho_{0}$ and $\varphi_{1}=\varphi_{2}=\varphi_{0}$. The first-order Taylor expansions of the vectors of interests are considered as follows:

$$
\begin{aligned}
\mathbf{b}\left(u_{1}\right) & =\mathbf{b}\left(u_{c}\right)-\frac{\delta}{2} \dot{\mathbf{b}}\left(u_{c}\right) \text { at }\left(u_{1}=u_{c}-\frac{\delta}{2}, \rho_{0}, \varphi_{0}\right), \\
\mathbf{b}\left(u_{2}\right) & =\mathbf{b}\left(u_{c}\right)+\frac{\delta}{2} \dot{\mathbf{b}}\left(u_{c}\right) \text { at }\left(u_{2}=u_{c}+\frac{\delta}{2}, \rho_{0}, \varphi_{0}\right), \\
\mathbf{b}(\hat{u}(t)) & =\mathbf{b}\left(u_{c}\right)+\gamma(t) \delta \dot{\mathbf{b}}\left(u_{c}\right) \text { at }\left(u^{\prime}(t)=u_{c}+\gamma(t) \delta, \rho_{0}, \varphi_{0}\right)
\end{aligned}
$$

in which the first-order derivative w.r.t. $u_{c}$ of vector $\mathbf{b}\left(u_{c}\right)=$ $\mathbf{p}\left(\rho_{0}, \varphi_{0}\right) \otimes \mathbf{a}\left(u_{c}\right)$ is defined as $\dot{\mathbf{b}}\left(u_{c}\right)=\mathbf{p}\left(\rho_{0}, \varphi_{0}\right) \otimes \dot{\mathbf{a}}\left(u_{c}\right)$ where $\dot{\mathbf{a}}\left(u_{c}\right)=j \operatorname{diag}\{\mathbf{d}\} \mathbf{a}\left(u_{c}\right)$. So, the optimal value (7) becomes

$$
\hat{s}(t) \cong p(t)+\frac{\delta}{N} \kappa_{c} m(t)
$$

in which $p(t)=s_{1}(t)+s_{2}(t), \kappa_{c}=\mathbf{b}^{H}\left(u_{c}\right) \dot{\mathbf{b}}\left(u_{c}\right), \mathbf{m}=$ $[m(1) \ldots m(T)]^{T}=\mathbf{V}^{T} \mathbf{s}$ where $\mathbf{s}=\left[s_{1}(1) s_{2}(1) \ldots s_{1}(T) s_{2}(T)\right]^{T}$ and $\mathbf{V}=\operatorname{Bdiag}\{\mathbf{v}(1), \ldots, \mathbf{v}(T)\}$ with $\mathbf{v}(t)=\left[\gamma(t)+\frac{1}{2} \gamma(t)-\right.$ $\left.\frac{1}{2}\right]^{T}$. According to the previous expression, we can see that the optimal source $\hat{s}(t)$ is approximated by a linear combination of the sources $s_{1}(t)$ and $s_{2}(t)$. Consequently, using the above expression the first-order Taylor expansion of the mean under $\mathcal{H}_{0}$ and $\mathcal{H}_{1}$ can be rewritten as

$$
\begin{aligned}
& \boldsymbol{\mu}_{0}(t)=\mathbf{b}(\hat{u}(t)) \hat{s}(t) \cong \delta \boldsymbol{\nu}_{0}(t), \\
& \boldsymbol{\mu}_{1}(t)=\sum_{k=1}^{2} \mathbf{b}\left(u_{k}\right) s_{k}(t) \cong \delta \boldsymbol{\nu}_{1}(t)
\end{aligned}
$$

where

$$
\begin{aligned}
& \boldsymbol{\nu}_{0}(t)=p(t) \gamma(t) \dot{\mathbf{b}}\left(u_{c}\right)+\left(\frac{\kappa_{c} m(t)}{N}\right) \mathbf{b}\left(u_{c}\right) \\
& \boldsymbol{\nu}_{1}(t)=\frac{q(t)}{2} \dot{\mathbf{b}}\left(u_{c}\right) .
\end{aligned}
$$

in which $q(t)=s_{2}(t)-s_{1}(t)$. So, the linearized hypothesis test is given by

$$
\begin{cases}\mathcal{H}_{0}: & \mathbf{z} \cong \delta \boldsymbol{\nu}_{0}+\mathbf{n} \\ \mathcal{H}_{1}: & \mathbf{z} \cong \delta \boldsymbol{\nu}_{1}+\mathbf{n}\end{cases}
$$

where $\mathbf{z}=\left[\mathbf{z}(1)^{T} \ldots \mathbf{z}(T)^{T}\right]^{T}, \mathbf{n}=\left[\mathbf{n}(1)^{T} \ldots \mathbf{n}(T)^{T}\right]^{T}, \boldsymbol{\nu}_{0}=$ $\left[\boldsymbol{\nu}_{0}^{T}(1) \ldots \boldsymbol{\nu}_{0}^{T}(T)\right]^{T}$ and $\boldsymbol{\nu}_{1}=\left[\boldsymbol{\nu}_{1}^{T}(1) \ldots \boldsymbol{\nu}_{1}^{T}(T)\right]^{T}$.

\section{ARL BASED ON INFORMATION THEORY}

\subsection{Stein's lemma}

By maximizing the probability of detection (i.e. $P_{d} \approx 1$ ) for $P_{f a} \leq$ $\epsilon$ with $\epsilon$ goes to zero slowly, the best error exponent resulting from using Neyman-Pearson test is given by the Stein's lemma $[11,12]$ as follows:

$$
\lim _{T \rightarrow \infty} \ln P_{f a}=-\mathcal{D}\left(p\left(\mathbf{z} \mid \mathcal{H}_{1}\right) \| p\left(\mathbf{z} \mid \mathcal{H}_{0}\right)\right)
$$

\footnotetext{
${ }^{1}$ Note that this situation is the worst case in the resolution point of view.
} 
where $\mathcal{D}\left(p\left(\mathbf{z} \mid \mathcal{H}_{1}\right) \| p\left(\mathbf{y} \mid \mathcal{H}_{0}\right)\right)$ denotes the relative entropy. Let $\Omega$ be the observation space. The relative entropy between two Gaussian distributions with parameterized means is given by:

$$
\begin{aligned}
\mathcal{D}\left(p\left(\mathbf{z} \mid \mathcal{H}_{1}\right) \| p\left(\mathbf{z} \mid \mathcal{H}_{0}\right)\right) & =\int_{\Omega} p\left(\mathbf{z} \mid \mathcal{H}_{1}\right) \ln \left(\frac{p\left(\mathbf{z} \mid \mathcal{H}_{1}\right)}{p\left(\mathbf{z} \mid \mathcal{H}_{0}\right)}\right) d \mathbf{z} \\
& =\frac{1}{\sigma^{2}} \sum_{t=1}^{T}\left\|\boldsymbol{\mu}_{0}(t)-\boldsymbol{\mu}_{1}(t)\right\|^{2} .
\end{aligned}
$$

\subsection{Geometrical expression of the relative entropy}

Using relations (10) and (11), we can link the relative entropy and the ARL according to

$$
\begin{aligned}
\mathcal{D}\left(p\left(\mathbf{y} \mid \mathcal{H}_{1}\right) \| p\left(\mathbf{y} \mid \mathcal{H}_{0}\right)\right) & \cong \frac{\delta^{2}}{\sigma^{2}} \sum_{t=1}^{T}\left\|\boldsymbol{\nu}_{0}(t)-\boldsymbol{\nu}_{1}(t)\right\|^{2} \\
& =\frac{\delta^{2}\|\mathbf{m}\|^{2}}{\sigma^{2}}\left\|\frac{\kappa_{c}}{N} \mathbf{b}\left(u_{c}\right)-\dot{\mathbf{b}}\left(u_{c}\right)\right\|^{2} \\
& =\frac{\delta^{2}\|\mathbf{m}\|^{2}\|\mathbf{d}\|^{2}}{\sigma^{2}} \cos ^{2}(\Theta)
\end{aligned}
$$

using $\|\mathbf{p}(\rho, \varphi)\|^{2}=1,\left\|\mathbf{a}\left(u_{c}\right)\right\|^{2}=N,\left\|\dot{\mathbf{a}}\left(u_{c}\right)\right\|^{2}=\|\mathbf{d}\|^{2}$ and $\Theta$ is the largest canonical angle between vectors $\mathbf{a}\left(u_{c}\right)$ and $\dot{\mathbf{a}}\left(u_{c}\right)$. The important point is that the relative entropy can be approximated by a quadratic (in $\delta$ ) expression. In addition, the relative entropy is a function of the source waveforms, of the array distribution, of the noise variance and of the a useful geometrical quantity which is the "angle" between the steering vector and its first-order derivative. It is interesting to note that the minimal value of $1 / \cos ^{2}(\Theta)$ which is reached for collinear vectors (i.e. $\Theta=0$ ) is not relevant since by construction the steering vector and its first-order derivative cannot be collinear. Another geometrical interpretation is: the more orthogonal the two vectors, the smaller the relative entropy. This means that it could be more and more difficult to discriminate the two hypothesis. According to the expression of the relative entropy, we can see that to ensure a "good" discrimination of the two hypothesis, we must have a large ARL or/and a large array distribution and/or a small noise variance.

\subsection{ARL based on the Stein's lemma}

Thus, from the above expressions and using the fact that for optimal $P_{d}$ (close to one), $P_{f a} \approx 2 P_{e}=2(1-\eta)$, the ARL takes a closedform expression according to

$$
\delta \cong \frac{-\sigma \sqrt{\log (2)+\log (1-\eta)}}{\mu\|\mathbf{d}\| \cos (\Theta)}
$$

where $\eta>1 / 2, \mu=\sqrt{\mathbf{s}^{H} \mathbf{V} \mathbf{V}^{T} \mathbf{s}}$ in which

$$
\mathbf{V} \mathbf{V}^{T}=\operatorname{Bdiag}\{\mathbf{G}(1) \ldots \mathbf{G}(T)\}
$$

where

$$
\mathbf{G}(t)=\left[\begin{array}{cc}
\left(\gamma(t)+\frac{1}{2}\right)^{2} & \gamma^{2}(t)+\frac{1}{4} \\
\gamma^{2}(t)+\frac{1}{4} & \left(\gamma(t)-\frac{1}{2}\right)^{2}
\end{array}\right] .
$$

It is interesting to note that the ARL is affected by the waveform design, $(c f$. quantity $\mu)$ but not from the polarization state in case of unit norm of the polarization vector.

\section{DETECTION THEORY APPROACH}

In this Section, we derive the ARL using the detection theory approach, particularly, using the well-known Neyman-Pearson (NP) criterion in the Bayesian context, denoted by BNP. It can be noted that the ARL in this context has not been derived in the existing literature. The BNP will minimize the probability of error $P_{e}$. Even if the proposed approach is Bayesian as the one presented by Amar and Weiss [8], our approach is different. Indeed, Amar and Weiss derive the ARL, denoted Theoretical Resolution Limit (TRL), based on the linearization of the error probability. In our method, we choose to linearize directly the observation signal as done by Sharman and Milanfard [10].

In order to simplify the calculation, we perform the following change of variable formula:

$$
\mathbf{z}^{\prime}=\frac{\mathbf{z}}{\delta}-\nu_{0}
$$

Consequently, plugging (18) into (14), one obtains

$$
\begin{cases}\mathcal{H}_{0}: & \mathbf{z}^{\prime} \cong \mathbf{n}^{\prime} \\ \mathcal{H}_{1}: & \mathbf{z}^{\prime} \cong \boldsymbol{\zeta}+\mathbf{n}^{\prime}\end{cases}
$$

where $\boldsymbol{\zeta}=\boldsymbol{\nu}_{1}-\boldsymbol{\nu}_{0}$ and $\mathbf{n}^{\prime} \sim \mathcal{C N}\left(\mathbf{0}, \frac{\sigma^{2}}{\delta^{2}} \mathbf{I}\right)$. Consequently, one has

$$
G_{N P}\left(\mathbf{z}^{\prime}\right)=\frac{p\left(\mathbf{z}^{\prime} \mid \mathcal{H}_{1}\right)}{p\left(\mathbf{z}^{\prime} \mid \mathcal{H}_{0}\right)} \underset{\mathcal{H}_{0}}{\stackrel{\mathcal{H}_{1}}{\gtrless}} \tau^{\prime}=\frac{p\left(\mathcal{H}_{0}\right)}{p\left(\mathcal{H}_{1}\right)},
$$

denoting $T_{N P}\left(\mathbf{z}^{\prime}\right)=\ln \left(G_{N P}\left(\mathbf{z}^{\prime}\right)\right)$ and $\tau=\ln \left(\tau^{\prime}\right)$, the statistics test can be given by

$$
\begin{aligned}
T_{N P}\left(\mathbf{z}^{\prime}\right) & =\ln \left(\frac{p\left(\mathbf{z}^{\prime} \mid \mathcal{H}_{1}\right)}{p\left(\mathbf{z}^{\prime} \mid \mathcal{H}_{0}\right)}\right)=\frac{\delta^{2}}{\sigma^{2}}\left(\left\|\mathbf{z}^{\prime}-\boldsymbol{\zeta}\right\|^{2}-\left\|\mathbf{z}^{\prime}\right\|^{2}\right) \\
& =\frac{\delta^{2}}{\sigma^{2}}\left(\|\boldsymbol{\zeta}\|^{2}-2 \Re\left\{\mathbf{a}^{H} \mathbf{z}^{\prime}\right\}\right) \underset{\mathcal{H}_{0}}{\stackrel{\mathcal{H}_{1}}{\gtrless}} \tau .
\end{aligned}
$$

Since we have assumed that $p\left(\mathcal{H}_{0}\right)=p\left(\mathcal{H}_{1}\right)=1 / 2$, one obtains

$$
\begin{cases}\mathcal{H}_{0}: & T_{N P}\left(\mathbf{z}^{\prime}\right)>0 \\ \mathcal{H}_{1}: & T_{N P}\left(\mathbf{z}^{\prime}\right)<0 .\end{cases}
$$

Let $L\left(\mathbf{z}^{\prime}\right)=\Re\left\{\boldsymbol{\zeta}^{H} \mathbf{z}^{\prime}\right\}$, one can easily obtain

$$
\begin{cases}\mathcal{H}_{0}: & L\left(\mathbf{z}^{\prime}\right) \sim \mathcal{N}\left(0, \varrho^{2}\right) \\ \mathcal{H}_{1}: & L\left(\mathbf{z}^{\prime}\right) \sim \mathcal{N}\left(\|\boldsymbol{\zeta}\|^{2}, \varrho^{2}\right)\end{cases}
$$

where

Thus [13],

$$
\varrho^{2}=\frac{\sigma^{2}\|\boldsymbol{\zeta}\|^{2}}{2 \delta^{2}}
$$

$$
P_{e}=\frac{1}{2}\left(\left(1-Q\left(\frac{-\|\boldsymbol{\zeta}\|^{2}}{2 \sqrt{\varrho^{2}}}\right)\right)+Q\left(\frac{\|\boldsymbol{\zeta}\|^{2}}{2 \sqrt{\varrho^{2}}}\right)\right),
$$

in which $Q($.$) denotes the right-tail function of the probability func-$ tion for a Gaussian random variable with zero mean and unit variance. Since $Q\left(\frac{-\|\boldsymbol{\zeta}\|^{2}}{2 \sqrt{\varrho^{2}}}\right)=1-Q\left(\frac{\|\boldsymbol{\zeta}\|^{2}}{2 \sqrt{\varrho^{2}}}\right)$, thus, one obtains

$$
P_{e}=Q\left(\frac{\|\zeta\|^{2}}{\sqrt{4 \varrho^{2}}}\right)
$$


Consequently, the ARL based on the BNP criteria is given by

$$
\delta \cong \frac{\sigma \sqrt{2} Q^{-1}(1-\eta)}{\mu\|\mathbf{d}\| \cos (\Theta)}
$$

where $Q^{-1}($.$) is the inverse of the right-tail function of the proba-$ bility function for a Gaussian random variable with zero mean and unit variance.

\section{SIMULATIONS RESULTS}

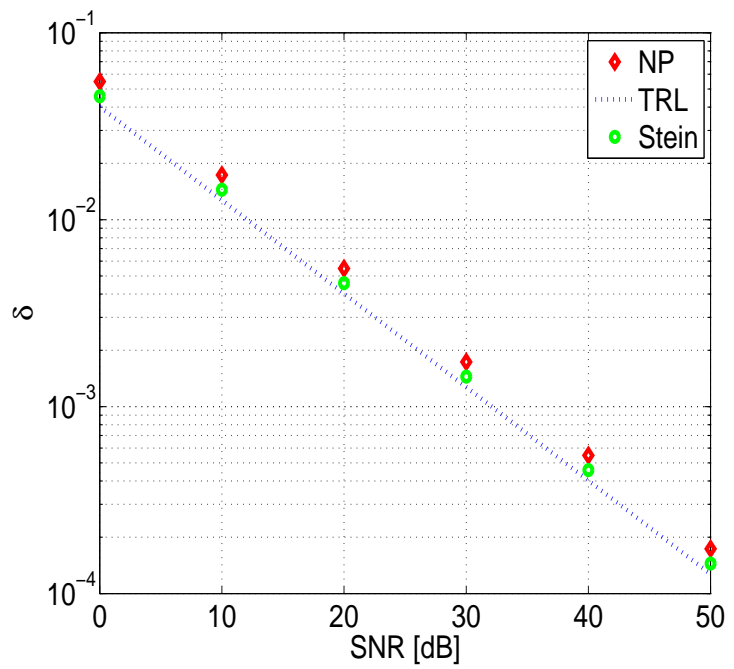

Fig. 1. ARL vs. SNR. The considered ARL are based on the Information Theory, on the Bayesian Neyman-Pearson (BNP), and on reference [8] denoted by TRL for Theoretical Resolution Limit.

In this section, we will compare the ARL based on the Information Theory, on the Bayesian Neyman-Pearson (BNP), and on reference [8] denoted by TRL for Theoretical Resolution Limit. We consider an uniform linear array consisting of $N=10$ vector-sensor components with a half-wavelength equidistantly space. The polarization parameters were chosen as $\rho_{0}=1$ and $\varphi_{0}=\pi / 3$. The success rate is $\eta=0.99$ thus $P_{e}$ is set to be 0.01 , and the probability of false alarm equals $P_{f a}=0.02$. We consider a large number of observations $T=100$. Since all parameters are herein assumed to be deterministic, the expectation concerning the TRL in [8], will not be performed. The signal to noise ratio $S N R$ is given by $S N R=\left(\sum_{k=1}^{2}\left\|\mathbf{s}_{k}\right\|^{2}\right) /\left(2 T \sigma^{2}\right)$. Fig. 1 shows the behavior of the ARL versus the SNR. One observes that the ARL based on the three methods are very close. It is interesting to note that the ARL is affected by the waveform design but not from the polarization state in case of unit norm of the polarization vector.

\section{CONCLUSION}

We have introduced, in this paper, a new approach based on the information theory to obtain the ARL for two closely-spaced polarized sources using a vector-sensor array. The key point is the fact that the relative entropy, in the Stein's lemma, can be approximated by a quadratic function in the ARL. So, it is possible to derive the ARL following this methodology. In addition, a geometrical expression of the ARL is provided. Finally, we compare our approach to the Bayesian Neyman-Pearson test (also derived in this paper) and to the Theoretical Resolution limit (TRL) proposed by Amar and Weiss. In particular, we show that for all these methods, the ARL is a function of the (unit) norm of the polarization vector but not of the specific values of the polarization parameters.

\section{REFERENCES}

[1] D. Rahamim, J. Tabrikian, and R. Shavit, "Source localization using vector sensor array in a multipath environment," IEEE Trans. Signal Processing, vol. 53, no. 11, pp. 3096-3103, Nov. 2004.

[2] S. Miron, N. L. Bihan, and J. I. Mars, "Vector-sensor MUSIC for polarized seismic sources localization," EURASIP Journal on Applied Signal Processing, vol. 2005, no. 1, pp. 74-84, 2005.

[3] S. Anderson and A. Nehorai, "Analysis of a polarized seismic wave model," IEEE Trans. Signal Processing, vol. 44, no. 2, pp. 379-386, Feb. 1996.

[4] A. Nehorai and E. Paldi, "Vector-sensor array processing for electromagnetic source localization," IEEE Trans. Signal Processing, vol. 42, no. 2, pp. 376-398, Feb. 1994.

[5] S. T. Smith, "Statistical resolution limits and the complexified Cramér-Rao bound," IEEE Trans. Signal Processing, vol. 53, no. 5, pp. 1597-1609, May 2005.

[6] M. N. El Korso, R. Boyer, A. Renaux, and S. Marcos, "Statistical resolution limits for multiple parameters of interest and for multiple signals," in Proc. IEEE Int. Conf. Acoust., Speech, Signal Processing, Dallas, TX, USA, Mar. 2010, pp. 3602 3605.

[7] K. Sharman and T. Durrani, "Resolving power of signal subspace methods for fnite data lengths," in Proc. IEEE Int. Conf. Acoust., Speech, Signal Processing, Florida, USA, 1995, pp. 1501-1504.

[8] A. Amar and A. J. Weiss, "Fundamental limitations on the resolution of deterministic signals," IEEE Trans. Signal Processing, vol. 56, no. 11, pp. 5309-5318, Nov. 2008.

[9] Z. Liu and A. Nehorai, "Statistical angular resolution limit for point sources," IEEE Trans. Signal Processing, vol. 55, no. 11, pp. 5521-5527, Nov. 2007.

[10] M. Shahram and P. Milanfar, "Imaging below the diffraction limit: A statistical analysis," IEEE Trans. Image Processing, vol. 13, no. 5, pp. 677-689, May 2004.

[11] T. Cover and J. A. Thomas, Elements of information theory. New York: Wiley, 1991.

[12] A. Jain, P. Moulin, M. Miller, and K. Ramchandran, "Information-theoretic bounds on target recognition performance based on degraded image data," IEEE Computer Society, vol. 24, no. 9, pp. 1153-1166, 2002.

[13] S. M. Kay, Fundamentals of Statistical Signal Processing. NJ: Prentice Hall, 1998, vol. 2. 